\title{
Navy Applications of High-Frequency Acoustics
}

\author{
Henry Cox
}

Lockeed Martin Orincon Defense, 4350 North Fairfax Drive, Suite 470, Arlington, VA 22202

\begin{abstract}
Although the emphasis in underwater acoustics for the last few decades has been in low-frequency acoustics, motivated by long range detection of submarines, there has been a continuing use of high-frequency acoustics in traditional specialized applications such as bottom mapping, mine hunting, torpedo homing and under ice navigation. The attractive characteristics of high-frequency sonar, high spatial resolution, wide bandwidth, small size and relatively low cost must be balanced against the severe range limitation imposed by attenuation that increases approximately as frequency-squared. Many commercial applications of acoustics are ideally served by high-frequency active systems. The small size and low cost, coupled with the revolution in small powerful signal processing hardware has led to the consideration of more sophisticated systems. Driven by commercial applications, there are currently available several commercial-off-the-shelf products including acoustic modems for underwater communication, multi-beam fathometers, side scan sonars for bottom mapping, and even synthetic aperture side scan sonar. Much of the work in high frequency sonar today continues to be focused on specialized applications in which the application is emphasized over the underlying acoustics. Today's vision for the Navy of the future involves Autonomous Undersea Vehicles (AUVs) and off-board ASW sensors. High-frequency acoustics will play a central role in the fulfillment of this vision as a means of communication and as a sensor. The acoustic communication problems for moving AUVs and deep sensors are discussed. Explicit relationships are derived between the communication theoretic description of channel parameters in terms of time and Doppler spreads and ocean acoustic parameters, group velocities, phase velocities and horizontal wavenumbers. Finally the application of synthetic aperture sonar to the mine hunting problems is described.
\end{abstract}

\section{INTRODUCTION}

Most World War II sonars operated in the 15 to $30 \mathrm{kHz}$ frequency range and would be considered "high frequency" today. Long range detection of submarines has been the primary concern that has motivated Navy sponsored research and development for the last several decades. This has led to an emphasis on low-frequency underwater acoustics. During this period there has been a continuing interest in high-frequency acoustics for special applications. The attenuation of sound in sea water is approximately $1 \mathrm{~dB} / \mathrm{km}$ at a frequency of $10 \mathrm{kHz}$ and increases as frequency-squared so that attenuation is about $4 \mathrm{~dB} / \mathrm{km}$ at $20 \mathrm{kHz}$. This severe attenuation limits the domain of applicability of high-frequency underwater acoustics to relatively short range. There are, however, many advantages to high frequencies for short range applications including high spatial resolution, wide bandwidth, small size and low cost. These characteristics are attractive for many commercial applications of underwater acoustics. The availability of low cost powerful computing has led to commercial systems of increased sophistication. The result is that the Navy is not the only major user of high-frequency underwater systems. Much of the work involving 
high-frequency underwater systems is focused on the particular application rather than basic research in the underlying acoustics. Navy applications include fathometers, mine hunting and avoidance, under ice navigation, torpedo homing, bottom mapping and communication. Fathometers, bottom-mapping sonars, and communication systems have a sufficient commercial market that they are advertised on the world wide web where specifications and pictures are presented.

Today, there is a new vision for the Navy that involves use of off-board sensors for Anti-Submarine Warfare (ASW) and Autonomous Undersea Vehicles (AUVs) for a number of missions. In these emerging systems, high-frequency acoustics will play a significant role for sensors and communications. Again the application interest predominates over the underlying acoustics.

\section{ACOUSTIC COMMUNICATIONS}

High-frequency acoustics is a key enabling technology for both AUV communication and AUV sensors. Today, unmanned Remotely Operated Vehicles (ROVs) are commonly used for inspection of oil rigs and similar commercial applications with very severe range restrictions. These vehicles are tethered to a mother platform with communications of nearly unlimited bandwidth via an optical fiber in the tether. With a submerged AUV there is no tether and no practical alternative to acoustic communications, except possibly at extremely short ranges. AUVs are envisioned as having multiple missions, involving different ranges and data rate requirements. Requirements for range and data rate drive the selection of frequency, bandwidth and power for an acoustic communications system. Desired data rates and ranges may or may not be compatible with a practical implementation. Thus, there will be a need to compromise between what is (really) required and what is feasible. In addition, in military applications there will be a premium placed on communication reliability, with an associated reduction in data rate to increase reliability. It is reasonable to consider multiple communication modes using different powers and different frequencies to achieve different data rates at different ranges and perhaps different channel conditions.

A complicated communication channel with multi-path and temporal variability is frequently characterized by two parameters: the time-spread $\tau$ and the Doppler-spread $\mathrm{B}$ or their reciprocals: the fading bandwidth $\mathrm{W}$ and the coherence time $\mathrm{T}$ [1]. Uncompensated time-spread limits the coherent processing bandwidth $\mathrm{W}$ to a value $1 / \tau$. Similarly temporal variability limits the coherent processing time $T$ to $1 / B$. The amount of coherent processing gain that the channel can support is $\mathrm{TW}$ or $1 / \mathrm{B} \tau$. For underwater communication applications with moving sources and receivers, such as when the AUV must be free to move with respect to the mother platform during communications, the temporal instability may depend more on source-receiver motion that on the temporal variability of the ocean. The motion converts spatial variability to temporal variability. For the situation of motion induced temporal variability; we can express the communication characteristics, $B$ and $\tau$ in terms of ocean acoustic parameters. 
Consider the situation of two multi-paths of comparable strength so that they interfere. The time spread between the two components is simply the difference in travel time from source to receiver. This can be expressed in terms of range $\mathrm{R}$ and the difference in the reciprocals of the average group velocities $\delta(1 / \mathrm{u})$ as follows:

$$
\tau=\mathrm{R} \delta(1 / \mathrm{u})
$$

An isospeed approximation is sometimes useful in shallow water when the range is much greater than the water depth. For horizontal propagation in an isospeed channel, the group velocity of a ray can be expressed in terms of the sound speed $\mathrm{c}$ and the grazing angle or ray angle $\theta$.

$$
\mathrm{u}=\mathrm{c} \cos \theta
$$

Then

$$
\tau=\mathrm{R} \delta(\cos \theta) /\left(\mathrm{c} \cos \theta_{1} \cos \theta_{2}\right) \approx \mathrm{R}\left[\theta_{2}^{2}-\theta_{1}^{2}\right] / 2 \mathrm{c}
$$

The small angle approximation is used to express results directly in terms of ray angles. For example, two rays with ray angles of 0 and 10 degrees ( 0.174 radians) produce a time-spread of about $1.5 \%$ of the propagation delay or $10 \mathrm{~ms} / \mathrm{km}$. If the angles were 0 and 20 degrees, the time spread would increase by a factor of four to $6 \%$ or $40 \mathrm{~ms} / \mathrm{km}$.

The radial coherence length or spatial scale in range $\Delta \mathrm{R}$ of the resulting interference pattern can be expressed in terms of the difference in horizontal wavenumbers $\Delta \mathrm{k}$ or equivalently the difference in the reciprocals of the phase velocities $\delta\left(1 / \mathrm{c}_{\mathrm{p}}\right)$ as follows:

$$
\Delta \mathrm{R}=2 \pi / \Delta \mathrm{k}=1 /\left[\mathrm{f} \delta\left(1 / \mathrm{c}_{\mathrm{p}}\right)\right]=\lambda / \delta(\cos \theta) \approx 2 \lambda /\left[\theta_{2}^{2}-\theta_{1}^{2}\right]
$$

At longer ranges in shallow water, higher modes or equivalently steeper angles may be stripped away due to multiple lossy reflections off the ocean bottom. Then, the steepest propagating angle and $\delta(\cos \theta)$ decrease slowly with range. This change with range is much slower than the interference induced change so that (4) remains valid as long as the correct interfering components are used. The coherence time is obtained by dividing this distance by the range rate $\mathrm{v}$.

$$
\mathrm{T}=1 /\left[\mathrm{f} \delta\left(1 / \mathrm{c}_{\mathrm{p}}\right) \mathrm{v}\right]=\lambda /[\delta(\cos \theta) \mathrm{v}]
$$

For the example of 0 and 10 degree rays, the coherence length is equal to 67 wavelengths. If the angles were 0 and 20 degrees this would reduce to about 17 wavelengths. At a frequency of $10 \mathrm{kHz}$, these coherence lengths become $10 \mathrm{~m}$ and 2.5 $\mathrm{m}$ respectively. At a 3 knot range rate, the coherence times become $6.7 \mathrm{~s}$ and $1.7 \mathrm{~s}$ respectively.

We can use (1) and (5) to express the product of Doppler-spread and time-spread in terms of ocean acoustic parameters. 


$$
\mathrm{B} \tau=\operatorname{Rfv} \delta\left(1 / \mathrm{c}_{\mathrm{p}}\right) \delta(1 / \mathrm{u}) \approx \mathrm{R} f \mathrm{v}\left[\theta_{2}^{2}-\theta_{1}^{2}\right]^{2} / 4 \mathrm{c}^{2}
$$

Small values of $\mathrm{B} \tau$ are good. For the previous examples, at a range of $10 \mathrm{~km}$, the values of $\mathrm{B} \tau$ are 0.015 and 0.24 . Notice that doubling the steepest angle from 10 to 20 degrees caused a factor of 16 increase in the $\mathrm{B} \tau$ product. The $\mathrm{B} \tau$ product is directly proportional to range, range-rate and frequency and for the isospeed case approximately proportional to the square of $\delta(\cos \theta)$ or the fourth power of the steepest angle. These dependencies plus the dependence of attenuation on frequency-squared frame the problem of system design for AUV acoustic communications.

If the channel impulse response were known, it could be taken into account in the signal design and the processing. Even if it were known, it would change after one coherence time interval. A number of communication techniques use probe signals and adaptive equalization algorithms that involve measuring and compensating for the channel properties in an attempt to overcome these limitations. These algorithms are an active area of research for the application to the acoustic channel. For the case in which the temporal variability of the channel is due to known radial motion, it may be possible to take the properties of the acoustic propagation into account in order to extend the domain of applicability of a channel measurement. Let $h(\tau ; R)$ be the measured impulse response at range $\mathrm{R}$. What is it at range $\mathrm{R}+\delta \mathrm{R}$ ? A first order approximation is

$$
\mathrm{h}(\mathrm{t} ; \mathrm{R}+\delta \mathrm{R})=\mathrm{h}(\mathrm{t}[1-\beta \delta \mathrm{R} / \mathrm{R}] ; \mathrm{R})
$$

The parameter $\beta$ is the wave guide invariant $[2,3]$. It is approximately unity for shallow water. The first approximation to the effect of a range increase is a stretch in the impulse response. This can be used to extend the useful life of a measurement when range and range rate are known [3, 4]. The inclusion of the physics of propagation into the techniques of adaptive channel equalization may give rise to improved practical approaches to acoustic communications between moving platforms.

For nearly stationary sources, motions that are on the order of a wavelength can cause loss of coherence. At $10 \mathrm{kHz}$ the wavelength is only six inches so that small motions are significant. Motion measurement and compensation can be used to improve performance. One approach is to use the phase of a known reference signal for compensation.

While the investigation of sophisticated processing techniques to maximize the efficiency of a communication system is a popular area of research, the designer of a practical system may opt for less sophistication and lower data rate in order to achieve robustness. Thus, an incoherent processing approach such as Multiple Frequency Shift Keying (MFSK) with redundancy coding may be chosen to provide reliable low data-rate communications in difficult environments. In AUV applications there may well be a need for multiple modes to accommodate the different range, data-rate and maneuvering requirements. For example, there might be a combination of a highfrequency, high data-rate, short range, coherent communication system and a long range, lower frequency, low data rate, incoherent MFSK system. 
While the requirements of some Navy systems may be special and appropriate for custom design, for other applications it would be desirable to have a "handbook", catalog, or MATLAB program that would allow tradeoffs in power, frequency, bandwidth, range and data-rate. The processing requirement for an acoustic communication system can be handled on a modern PC so that system hardware is not much more than a laptop computer, a transducer and a power amplifier. The cost can be kept reasonable for routine applications. The power amplifier and the computer can be common across a variety of applications so that a family of systems with different transducers at different frequencies can provide capability at different ranges and data-rates. A step in this direction is available in the commercial sector as can be seen by visiting the internet where a number of commercial acoustic modems are advertised.

The application of acoustic communications to off-board sensors is also of current Navy interest. For sensors the major tradeoff is between more processing in the sensor to reduce the communication data-rate and simpler in-sensor processing with more data being sent in the sensor reports. The sonobuoy has been a widely used off-board sensor for several decades. It uses radio communications. For some other sensors radio communications is also an attractive option.

An application in which acoustic communication is the only practical alternative is a Reliable Acoustic Path (RAP) acoustic receiver for either passive or active ASW. The concept is simple and serves as a specific example of a well posed design problem. The solution will be left to the reader. The acoustic receiver is deployed from an aircraft or ship and then sinks to the bottom in deep water with a depth of several thousand meters. The deep receiver location provides direct path acoustic coverage to a range of several water depths. The sensor is battery powered so that the power budget for communication is a concern. It reports via an acoustic link to a ship or sonobuoy that relays the data by radio the ultimate user. The acoustic link must function reliably over a path-length of up to $18 \mathrm{~km}$. The acoustic propagation is simple with the only multi-path arising from a bottom reflection at the reporting sensor and a surface reflection at the receiver. Modest directivity can be used to minimize the effects of these multi-paths. The grazing angle of the propagation is steep so that refraction is not significant. The ambient noise falls off with frequency at about $6 \mathrm{~dB}$ per octave. Some array gain against ambient noise can be provided in the receiving sonobuoy to reduce the power requirement. The sonobuoy relay is drifting and experiencing motion due to surface wave action. The acoustic propagation loss at of $18 \mathrm{~km}$ is about $85 \mathrm{~dB}$ plus frequency dependent absorption. The problem is to provide the trade-offs concerning frequency, power and data-rate that can feed into other total system design issues such a processing approach, battery and system life.

\section{SYNTHETIC APERTURE SONAR (SAS)}

While the use of Synthetic Aperture in Radar (SAR) is very mature, the sonar counterpart is in its early stage of development and application. It is an exiting application area for high-frequency acoustics. The basic idea is that coherently adding 
data from successive pings with a moving real side-looking aperture looking at a fixed scene is equivalent to observing the scene with a larger aperture.

The spatial sampling requirement is that the motion between pings should be less than one-have the length of the real aperture. The maximum unambiguous range is one-half the distance sound travels between pings. These two conditions restrict the search rate. One can either ping less frequently, move more slowly and work to longer range, or move more rapidly, ping more often and work to shorter range. The result is that the search rate is proportional to the real aperture-length not the speed of advance. The shape of the searched area depends on speed but the search rate does not. The speed of sound is relatively slow compared to the speed of light so that vehicles speeds are slow and it takes minutes to build a synthetic sonar image. An issue has been whether or not the sonar environment would be sufficiently stationary over the time it takes to build an SAS image. The answer seems to be yes. A set of practical difficulties are associated with motion compensation for deviations from constant linear motion by the array. Algorithms for motion compensation, similar to the ones used in radar have now been successfully applied in sonar. The signal processing for SAS has been made practical by the advent of small, cheap, powerful computers. Research systems have been built in several countries and in the US under sponsorship of DARPA and ONR. They have demonstrated impressive improvement in range and resolution over conventional side looking sonar.

The Navy application of most current interest is mine hunting. Increased swath width increases search-rate but more importantly the large increase in resolution provides finer shape information that greatly simplifies the problem of distinguishing mines from other objects on the bottom. Because in mine countermeasures no false alarm can go uninvestigated, a reduction in false alarms greatly reduces the time it takes to clear an area. An AUV with a SAS sensor is a particularly attractive concept for mine hunting in which high-frequency acoustics provides the fundamental enabling technology for both sensing and communications.

\section{CONCLUSION}

High-frequency acoustics has many applications to Navy problems. Two of the most technologically advanced are acoustic communications and SAS. These come together in the vision of the future Navy that involves AUVs and off-board sensors. The current interest seems to be more in the application than in the underlying acoustics and the research has a heavy signal processing flavor. The discussion of the communication problem presented for the first time explicit relationships between the signal processing quantities parameters of time and Doppler spread and the underlying ocean acoustic and physical quantities such as horizontal wavenumbers, group velocities, and phase velocities, range and range rate. 


\section{REFERENCES}

1. A.B. Baggeroer, "Acoustic Telemetry - An Overview", IEEE J. Oc. Acoustics,9 (4), 1984

2. G.L. D'Spain and W.A. Kuperman, "Application of waveguide invariants to analysis of spectrograms from shallow water environments that vary in range and azimuth", JASA, 106 (5), 1999

3. H. Cox and K. Heaney, "Interference Patterns in Range Dependent Environments", $144^{\text {th }}$ Meeting of the Acoustical Society of America, December 2002. JASA 112(5), 2002

4. H. Cox, H. Lai and M. Hirano, "Reciprocity Based Channel Compensation for Wideband Communication in a Multipath Environment", Proceedings of the 27th Asilomar Conference on Signals, Systems and Computers, Pacific Grove California Nov. 1-3, 1993. 